\title{
An Analysis of Chinese International Students' Acculturative Strategies in Western Universities
}

\author{
Ying Chen ${ }^{1, \dagger}$ Haoran $\mathrm{Wu}^{2, *}, \dagger$ \\ ${ }^{1}$ Guangdong Country Garden School, Foshan, Guangdong, China, 528000. \\ ${ }^{2}$ College of the Holy Cross, Worcester, Massachusetts, United States, 01610. \\ ${ }^{*}$ Corresponding author email: HWU22@g.holycross.edu \\ ${ }^{t}$ Those authors contributed equally.
}

\begin{abstract}
The acculturation of immigrants has contributed to the cultural diversity of a society or even a country. It is a complex process since it involves about two cultures or even more than two cultures. This work aims to review the process of acculturation of Chinese international students during their studies in the United States, and how various types of acculturations formed. Additionally, it also investigates the causes of the formation of these strategies, which can help Chinese international students know what leads them into this situation and what they can do to make a better of their life abroad. Here, a bi-dimensional model proposed by Berry identifies four specific acculturative strategies including integration, separation, assimilation, and marginalization, which are employed by the migrant individuals. This study concluded that the most important reason for the unsuccessful integration of Chines international students is language issues, for example, the inability to understand slang. In the future, we can propose different countermeasures at various levels, (e.g. government, social, school, and individual) to improve the situation of unsuccessful integration into the host nations.
\end{abstract}

Keywords: Acculturation, Chinese international students, assimilation, marginalization.

\section{INTRODUCTION}

When Chinese international students leave their motherland and study in an English-speaking foreign country, which has a different campus environment from China. During the period of living in a foreign country, they need to take time to adapt and get used to the new and different environment, this process is called acculturation, which occurs when Chinese international students contact nationals in the host country. Berry refers that in this process, individuals in the acculturative group can have a different experience from the general group, which means the local people [1]. To understand how various migrant populations have an adaptation, Berry proposes a bi-dimensional model to identify four types of acculturation, including integration, separation, assimilation, and marginalization [2]. How these four types of acculturation are formed and what are the reasons can cause these results would analysis deeply as well. As a result, it leads to a series of issues: how can Chinese international students realize they experience which strategies, which is passive or the strategy that can benefit them? Also, how they solve the problems when they experience passive strategies?

\section{THEORETICAL FRAMEWORK}

According to Redfield, Linton, and Herskovits, the term "acculturation" refers to "phenomena which result when groups of individuals having different cultures come into continuous first-hand contact with subsequent changes in the original culture patterns of either or both groups" [3]. When Chinese international students depart their home country and live in a foreign, Englishspeaking campus environment, they inevitably experience such first first-hand contact with nationals in the host country, and thus their acculturative process takes place in this interaction. Scholars such as Graves have made a distinguishment between phenomenal, group-level acculturation and the phycological, individual-level acculturation [4]. The latter is particularly crucial for understanding Chinese international students' acculturation because, as Berry points out, the individuals within the acculturative group 
can have different experiences and values compared to the ones held by the Chinese student group in general [1].

To better understand the phycological acculturative process of various migrant populations, Berry proposes a bi-dimensional model to identify four specific acculturative strategies employed by the migrant individuals [2]. The two dimensions are the degree where a migrant individual recognizes the importance of preserving his own values and identities and the degree where he recognizes the importance of incorporating the values and identities of the larger society [3]. If the individual is seeking values and identities of the larger society while also value their own cultural heritage, then integration is the term to describe such a strategy [3]. When the individual is preserving his own values and identities but is not incorporating the host society's ones, separation will occur in the acculturative process [3]. Alternatively, if he rejects his own values and identities but embraces the host society's ones, then he is taking the approach of assimilation [3]. Finally, if the individual accepts neither the values and identities of his own nor those of the host society, his strategy will be categorized into the term marginalization [3]

This bi-dimensional model of analyzing acculturative strategies has been employed by Berry to describe a fivestep approach of phycological acculturation $[2,5]$. The first step is the encounter of important life events, which demands acculturative individuals to mitigate the differences between their values and identities and the ones of the larger society $[2,5]$. For Chinese international students, some of the differences include the style of teaching, the style of learning, ways to socialize, opinions on religion, ideological affiliation, and more. In the second step, the acculturative individuals will "consider the meaning of these experiences, evaluating and appraising them as a source of difficulty (i.e. as stressors), or as benign, sometimes even as opportunities" $[2,5]$. If the individuals perceive these events as benign or opportunities, they will likely take a behavioral shift, leading to either integration or assimilation. [2, 5]If they perceive these life events as stressors that are moderately problematic or overwhelming, such behavioral shifts may not take place, more likely leading to separation and marginalization $[2,5]$. As the third step, the acculturative individual will employ the coping strategies mentioned above. In the fourth step, these strategies will produce various levels of stress, depending on whether or not behavioral shifts have occurred $[2,5]$. Finally, in the last step, the stress level will determine the nature of acculturative individuals' adaptation, in which ones with less stress will integrate effectively into the society, while ones with more stress will likely become isolated[2,5].

Berry's framework of acculturative strategies has been widely employed and developed into subsequent models [6, 7]. For instance, Piontkowski, Rohmann, and Florack propose the concordance model of acculturation
(CMA) [6]. They list four possible acculturative outcomes depending on whether dominant and nondominant groups share the same value on one or both of Berry's dimensions: consensual, cultural-problematic, contact-problematic, and conflictual [6]. Also, Navas et al. have developed the Relative Acculturation Extended Model (RAEM), in which they mention that acculturative individuals will choose one of the four strategies based on circumstances [7]. Specifically, in the work and political contexts, they found out that migrants are more likely to adopt assimilation, whereas, in the social context, migrants are more likely to choose integration [7]. Since Berry's acculturation framework is extensively discussed and expanded, studies have employed it to examine international students' acculturation strategies. For example, Sullivan and West's study has shown that international students are more likely to experience marginalization and subsequent acculturative stress than other migrant groups [8]. Nevertheless, few of these studies have focused specifically on Chinese students, which constitute the largest international student population in many English-speaking countries. Based on Berry's framework of the phycological acculturation process and strategies, this paper will address the following questions:

1) how often have these strategies been employed by Chinese international students?

2) In the case of integration and assimilation, how successful are these strategies in dealing with acculturation? On some occasions, why did they fail?

3 ) In the case of separation and marginalization, what stressors have compelled them to take these passive strategies?

\section{ACCULTURATIVE STRATEGIES OF CHINESE INTERNATIONAL STUDENTS}

\subsection{Integration}

As the most desirable method of acculturation, integration has been regarded by many Chinese international students, as the preferred strategy when they first enter a western, English-speaking campus and society. However, as numerous surveys, questionaries, interviews, and quantitative studies have suggested, the integration path contains many obstacles, and Chinese international students' level of success in integration is mixed. On the one hand, some students experience successful integration, in which they recognize the importance of preserving their own identities while also accepting the norms of mainstream society [9]. In a qualitative study of the relationship between Chinese international students' use of English names, assimilation, and self-presentation, Fang and Fine argues that for students who dropped their English names, their ethnic names serve as a representation of their unique 
Chinese identity compared to other groups in the host society [9]. Such demonstration can be viewed as a form of successful integration. Take the experience of Dawei, a Chinese international student who dropped his English name, David, as an example [9]:

"In college, I realized many Davids I knew are Jewish. I think David is a very Jewish name. Many Asians are called David, too, but if you don't count these Asians, most Davids in the US are Jewish. I don't want my name to have such cultural connotations... I had the idea that I needed to change my name...I am Chinese, not American."

In this case, the student demonstrated both of the features of acculturative integration. First, he acknowledges the norms and values of mainstream society and conforms to them. He understands that David is a name that many Jewish and Asian Americans have used, and he realizes that continuing the usage of the name "David" will violate such norms, leading to confusion. Second, the student possesses an intention of preserving his own cultural identities, in which he argues that he belongs to a distinctive "Chinese" population that is different from other populations in the society. More importantly, he implicitly recognizes uniqueness as one of the positive qualities that American mainstream culture values. Such recognition can be treated as a crucial step towards successful integration.

On the other hand, as studies have shown, Chinese international students have failed to integrate into their host society effectively in some other cases. Many factors have led to the difficulty of their social integration. Specifically, the lack of inclusiveness of host nationals is one of the more significant ones [10]. Berry argues that in order to make the integration of non-dominant groups successful, the dominant society must recognize the importance of cultural diversity, in which different cultural groups are allowed to maintain their identities and values [2]. Such recognition is not present in British universities, in which Oatey et al.'s survey of 2031 undergraduate and graduate students across the United Kingdom has indicated that some British student groups view Chinese international students critically, discouraging them from social integration[10]. Here is an example of a comment made by a female British student [10]:

"Without meaning to sound racist, the Chinese people in our flat never socialise with any of us. They don't even say hi when we do..."

This comment reveals some British students' implicit unacceptance of values and norms outside of their mainstream culture. Specifically, she believes the only method of demonstrating friendliness is to say hi. But in many cultures, waving hands, smiling, or nodding can also be viewed as amicable signs. If Chinese international students use these methods, British students may not interpret them as a symbol of friendliness because they do not accept cultural norms outside of their mainstream society.

In addition to the lack of inclusiveness, other factors may also undermine the social integration of Chinese international students $[10,11]$. For instance, the large size of the Chinese student population in some universities may limit their desire to socialize with host individuals outside of their confront zones [10]. Furthermore, some campuses' remoteness and lack of internship opportunities may prevent Chinese international students from engaging with host people of the society in general [10]. Also, some have pointed out that the loneliness of living in a foreign nation. As they are far away from their parents and families, they may choose to live and socialize with other international students, which will arguably undermine the chance of successful social integration with the host society [11]. Finally, as Oatey et al. suggest, personal characteristics play a pivotal role in determining the level of integration [10]. Chinese international students who are more outgoing, openminded, and extroverted are more likely to become socially integrated than others [10].

\subsection{Separation}

When Chinese international students found themselves living in a completely new environment, many of them would choose to separate themselves from the host society. Specifically, they primarily socialize with people within their group, and they also committed to retaining their Chinese lifestyle when living with each other. Berry has made a distinction between separation and the term segregation [2]. The former involves the active efforts of the non-dominant group to set a boundary between them and the dominant group, while the latter is a passive countermeasure against pressures from the dominant group [2]. Therefore, in the case of Chinese international students, their deliberate action of distancing themselves from the host society should be defined as separation rather than segregation.

Studies have shown that several stressors have compelled many Chinese international students to choose separation as their acculturative strategy [12,13]. One of them, and arguably the most influential one, is the desire to retain their Chinese cultural identity [12]. In Liu and Dong's research, they found out that all of their participants reject the possibility of abandoning their Chinese cultural heritage in exchange for better acculturative outcomes [12]. The following is a comment made by one of the Chinese respondents, Ying, on western and Chinese food [12]:

"I feel that many Americans are living a very pitiful life, especially those living in this small town. The food they eat is too unhealthy... All of these is because there is 
no way for them to eat the healthy food like Chinese. I personally still like Chinese food."

In this case, the student uses the word "pitiful" to show that she does not accept and appreciate American eating habit, which is a part of the mainstream norms. It is the decisive factor that distinguishes separation from integration, in which individuals who choose separation as their acculturative strategy do not recognize the importance of incorporating the host society's values and norms. Nevertheless, one can imagine that students who hold such rejective attitudes may have tried to incorporate mainstream norms such as having American food, and the failure to do so forced them to retreat from integration to separation.

Another stressor that frequently makes Chinese international students employ separation is the need to maintain their national pride under the increasing political tension between the western world and China [13]. In Zhao's study of Chinese international student's national identity, he discovered that they had attached their national identities to China either through the idea that they belong to where they were born or through the heritage of Chinese values such as resilience and diligence [13]. In addition, they often express their Chinese national identity by defending the country against criticisms [13]. These behaviors suggest that many Chinese international students have actively separated themselves away from the general population, unwilling to become homologous in terms of national identity.

\subsection{Assimilation}

The term "assimilation" has been extensively discussed in cross-cultural studies. While assimilation is often recognized as a synonym of acculturation, it is only one form of acculturative strategy, as the previous section of the paper has already explained. Similar to integration, a prerequisite of assimilation is that the out-group, or the host society, must fully identify the migrant population as an integral part of the community [14]. In terms of Chinese international students, with the fact that many of them are unwilling to abandon their Chinese identities, they are rarely successful in fully assimilating themselves into western societies [15]. There are numerous factors that have led to this outcome. The first one is the lack of English oral proficiency [15]. In Yan and Berliner's study, they found out that many Chinese international students are comfortable listening and talking in the academic and working environments, but when it comes to socializing with host nationals in private, they cannot keep up with native speakers' speed of conversation and usage of slangs [15]. Moreover, Chinese international students struggle to assimilate because of the lack of understanding of mainstream cultural knowledge [15]. One student in Yan and Berliner's study, for example, misinterprets an African American person's friendly greeting of "give me five" as a robbery, giving him five dollars [15]. As the oral language deficiency, such lack of cultural knowledge may not trouble Chinese international students in academic and working settings but does matter in social settings, in which their success in them is crucial to the assimilative process [15].

In addition, the different understanding of friendship often raises conflicts between Chinese international students and host nationals, leading to failure of assimilation in socialization [15]. For instance, a student in Yan and Berliner's study complains that one of his American friends has refused to help him proofread his paper for free and askes him for money in return [15]. In China, an authentic friendship means that individuals will help each other whenever they can during a crisis, but for the American host nationals, making contributions of time and effort in friendships does not differ from working in their jobs, and monetary rewards are expected [15]. Thus, many Chinese international students seeking to assimilate may not be able to adjust their attitude toward friendship according to the mainstream culture, leading to the failure of such assimilation.

\subsection{Marginalization}

As the most unfavorable acculturation approach, marginalization has often been interpreted as the result of the migrant individual's failure to assimilate successfully into the host society. Berry argues that when assimilation occurs, the migrant individual undergoes cultural shedding, the active removal of its original cultural behavior, and cultural learning, the acquiring of new behaviors that can fit into the new society [2]. However, when one experienced cultural shedding but not cultural learning, Berry believes, it will lead to marginalization, in which the individual does not value both the culture of its own and the culture of the host society [2].

Thus, the stressors that lead to Chinese international students' marginalization and higher acculturative stress are arguably derived from the obstacles of assimilation. When these obstacles, such as language and cultural barriers mentioned in the previous section, are perceived as daunting, many Chinese international students will decide to retreat into marginalization. They refuse to accept the host society's culture while also denying their culture developed in China. For instance, Fan and Maliborska's study provides the case of Lin, a first-year Chinese international student studying in the US [16]. When Lin was in high school, she was enthusiastic about becoming assimilated into the American culture [16]. After she came to the University campus, she attempted to socialize with the American students by living in a dorm away from other Chinese international students and participate in a part-time job at the food court [16]. However, due to her severe lack of listening and speaking skills, she not only struggled to socialize with American peers in the dorm and during the newcomers welcoming 
activity but also failed to communicate with her colleague in part-time jobs [16]. Lin's experience reflects how marginalization can occur in some Chinese international students' failed attempts to assimilate. First, due to their preconceived image of American culture and perceived easiness of assimilation, they abandon their conational network similar to Lin at the early stage of the assimilative process. As difficulties emerge because of significant language and cultural barriers, they realize that the prospect of successful assimilation is grim, and at the same time, separation is not an option for them because they already cut off their ties with other Chinese international students. Therefore, they can only choose to marginalize themselves, leading to the highest level of acculturative stress compared to stress brought by other strategies.

In addition to marginalization in the social context, some Chinese international students also fall into a marginalized category in the academic environment. Valdez's study describes this phenomenon as "double consciousness," in which some Chinese international students implicitly identify themselves as Chinese in classrooms but also disassociate themselves from stereotypes made by American faculties and classmates on Chinese students [17]. Specifically, on the one hand, Valdez discovers some of the Chinese international students complain that class activities in American classrooms presuppose them to have strong verbal skills and a deep understanding of American popular culture [17]. Such complaint indicates themselves as a distinct student body that differs from their American peers [17]. On the other hand, Valdez's study also shows many Chinese international students believe that American faculties and students may have negative views of them [17]. Many of them claim that their faculties would increase their scrutinization of Chinese students during exams because of the stereotype of Chinese students as cheaters, and American classmates would view them as uncooperative members of group works [17]. The fact that some Chinese international students emphasize their characteristics as Chinese and disassociate themselves from Chinese stereotypes simultaneously indicates how they have become marginalized in classrooms. In this process, they do not fully value both Chinese and American classroom norms.

\section{CONCLUSION}

To sum up, this paper discusses the four-level of acculturation when Chinese international students leave their home country and live in a foreign campus environment. There are a variety of reasons that cause these four specific strategies for both successful and failure examples. The main findings of this paper are:1) for integration, which is a preferred method for most the Chinese international students. The reasons for the people who integrate effectively due to they recognize the importance of how to maintain their own values and identities and accept mainstream society. However, some Chinese international students could not succeed in integrating into the host society. The lack of inclusiveness of host nationals contributes some international students' failures to integrate. 2) most of the Chinese international students reject to abandon their Chinese cultural heritage to exchange for better acculturative outcomes, the growing political tensions between the Western world and China, leading to the need for Chinese students to assert their national pride, so they would choose separation rather than integration. 3) Assimilation, Chinese international students are rarely succeeded in this strategy since China is the place where everybody has lived for many years, and Chinese culture has long been deeply engraved in everyone's bones. They hardly abandon their Chinese values and identities. 4) When it comes to marginalization, some Chinese international students may consider that assimilation can be achieved easily due to their preconceived impressions of American culture. However, during the process of assimilation, they recognize it difficult to assimilate successfully. At the same time, they did not contact the Chinese international students. Therefore, they have no choice but to experience marginalization.

\section{REFERENCES}

[1] J.W. Berry, Marginality, stress and ethnic identification in an acculturated Aboriginal community. Journal of Cross-Ciilrural Psychology 1 (1970) 239-252.

[2] J.W. Berry, Immigration, Acculturation, and Adaptation. Applied Psychology: An International Review 46 (1997) 5-68.

[3] R. Redfield, R. Linton, M. Herskovits, Memorandum on the study of acculturation. American Anthropologist 38 (1936) 149-152.

[4] T. Graves, Psychological Acculturation in a TriEthnic Community. Southwestern Journal of Anthropology 23 (1967) 337-350.

[5] D. Sam, J.W. Berry, The Cambridge Handbook of Acculturation Psychology, second edition., Cambridge University Press, Cambridge, 2016.

[6] U. Piontkowski, A. Rohmann, A. Florack, Concordance of Acculturation Attitudes and Perceived Threat. Group Processes \& Intergroup Relations 5 (2002) 221-232.

[7] Navas et al., Acculturation strategies and attitudes according to the Relative Acculturation Extended Model (RAEM): The perspectives of natives versus immigrants. International Journal of Intercultural Relations 31 (2007) 67-86. 
[8] C. Sullivan, S. Kashubeck-West, The Interplay of International Students' Acculturative Stress, Social Support, and Acculturation Modes. Journal of International Students 5 (2015) 1-11.

[9] J. Fang, G.A. Fine, Names and Selves: Transnational Identities and Self-Presentation among Elite Chinese International Students. Qualitative Sociology 43 (2020) 427-448.

[10] H. Spencer-Oatey et al., Chinese students' social integration into the university community: hearing the students' voices. High Educ 74 (2017) 739-756.

[11] G. Zhou, Z. Zhang, A Study of the First Year International Students at a Canadian University: Challenges and Experiences with Social Integration. Canadian and International Education 43 (2014).

[12] Y. Liu, Y Dong, Shared Experiences and Resilience of Cultural Heritage: Chinese Students' Social Interaction with Non-Host Nationals in the United States. Journal of International Students (2019) 111128.

[13] K. Zhao, Made in contemporary China: exploring the national identity of Chinese international undergraduate students in the US. Studies in Higher Education 45 (2020) 2451-2463.

[14] R.H.C. Teske, B.H. Nelson, acculturation and assimilation: a clarification. American Ethnologist 1 (1974) 351-367.

[15] K.Yan, D.C. Berliner, Chinese International Students' Personal and Sociocultural Stressors in the United States. Journal of College Student Development 54 (2013) 62-84.

[16] Y.S. Fan, V. Maliborska, Birds of a Feather Flock Together?---A Case Study on Socialization Experiences of Chinese International Student in an American University. International Journal of TESOL Studies 1 (2019) 43-70.

[17] G. Valdez, U.S. Higher Education Classroom Experiences of Undergraduate Chinese International Students. Journal of International Students 5 (2015) 188-200. 